\title{
THE AVERAGE OF THE RECIPROCAL OF A FUNCTION
}

J. ERNEST WILKINS, JR.

1. Introduction. Let $f(x)$ be an integrable function defined on an interval $(a, b)$. Its average value is

$$
A(f)=\frac{1}{b-a} \int_{a}^{b} f(x) d x .
$$

There are circumstances $[1 ; 2]$ in which one wishes to calculate not $A(f)$ but

$$
B(f)=\left[A\left(f^{-1}\right)\right]^{-1}=(b-a) / \int_{a}^{b} \frac{d x}{f(x)},
$$

although $A(f)$ is much easier to calculate. If $f(x)$ is constant, $A(f)$ $=B(f)$, and it is reasonable to expect that $A(f)$ will be approximately equal to $B(f)$ if $f(x)$ does not vary too widely. We propose to determine here the extreme values of the ratio

$$
I(f)=A(f) / B(f)
$$

as $f$ varies over a special class of functions.

Suppose that $0<\alpha<\beta$, and that $\mathfrak{A}$ is the class of measurable functions $f(x)$ defined on $(a, b)$ for which $\alpha \leqq f(x) \leqq \beta$. It is a consequence of a result of Pólya and Szegö [3] that

$$
1 \leqq I(f) \leqq(\alpha+\beta)^{2} / 4 \alpha \beta
$$

when $f(x)$ is in $\mathfrak{A}$. If $\mathfrak{B}$ is the class of concave (i.e., arc lies above chord) monotone decreasing functions $f(x)$ in $\mathfrak{A}$ which assume the values $\alpha$ and $\beta$, then we shall prove the better result that

(2) $1 \leqq I(f) \leqq \beta\left[\frac{\beta \ln (\beta / \alpha)}{\beta-\alpha}-\frac{\beta+\alpha}{2 \beta}\right]^{2} / 2(\beta-\alpha)\left[\frac{\beta \ln (\beta / \alpha)}{\beta-\alpha}-1\right]$.

These same bounds apply if $f(x)$ is a concave, monotone function in $\mathfrak{A}$, since the transformation $x^{\prime}=a+b-x$ converts increasing functions into decreasing functions without altering concavity or bounds, and since the right-hand side of (2) is a strictly increasing function of $\beta / \alpha$.

2. Existence of a maximizing function in $\mathfrak{B}$. Our proof of the in-

Received by the editors August 26, 1954 and, in revised form, December 9, 1954. 
equality (2) consists of a demonstration of the existence of a function $f_{0}(x)$ in $\mathfrak{B}$ for which $I(f)$ attains its least upper bound on $\mathfrak{B}$, followed by the deduction of various properties which any such maximizing function must possess. There will be precisely one function in $\mathfrak{B}$ possessing these properties and so it must be the maximizing function.

THEOREM 1. There exists a function $f_{0}(x)$ in $\mathfrak{B}$ for which $I(f)$ is a maximum.

We begin by observing that when $f$ is concave, there exists a function $f^{\prime}(x)$ which is decreasing and integrable on $(a, b)$ such that

$$
f(x)=f(a+)+\int_{a}^{x} f^{\prime}(t) d t, \quad a<x<b .
$$

The set of discontinuities of $f^{\prime}(x)$ on the open interval $(a, b)$ is a finite or denumerable set $E(f)$ and so $f^{\prime}(x)$ is the derivative of $f(x)$ except on $E(f)$ and possibly at $a$ and $b$. In addition when $f(x)$ is in $\mathfrak{B}$, $f^{\prime}(x) \leqq 0$. Moreover $f(x)$ is continuous when $a \leqq x<b$, and $f(b-) \geqq f(b)$ $=\alpha$.

LEMmA 1. If $f(x)$ is in $\mathfrak{B}$ and $c=b-M^{-1}(\beta-\alpha)>a$, then $f^{\prime}(x) \geqq-M$ when $a \leqq x \leqq c$.

For suppose there were a point $y$ such that $a \leqq y \leqq c, f^{\prime}(y)<-M$. Then, since $f^{\prime}(x)$ is decreasing, $f^{\prime}(t)<-M$ when $y \leqq t \leqq b$. Hence

$\beta \geqq f(y)=f(b-)-\int_{y}^{b} f^{\prime}(t) d t>\alpha+(b-y) M \geqq \alpha+(b-c) M=\beta$, and this is impossible.

Let $\mu$ be the least upper bound of $I(f)$ on $\mathfrak{B}$, and pick a sequence $f_{n}(x)$ of functions in $\mathscr{B}$ for which $\mu=\lim I\left(f_{n}\right)$. These functions may be chosen as continuous. Pick a monotone increasing sequence of numbers $M_{k}$ such that

$$
a<c_{k}=b-M_{k}^{-1}(\beta-\alpha) \rightarrow b .
$$

According to Lemma $1,0 \geqq f_{n}^{\prime}(x) \geqq-M_{k}$ when $a \leqq x \leqq c_{k}$, and so the functions $f_{n}(x)$ are equicontinuous and uniformly bounded on the closed interval $\left(a, c_{k}\right)$. By Ascoli's theorem, there exists a subsequence of the sequence $f_{n}(x)$ which converges uniformly to a limit $g_{1}(x)$ on $\left(a, c_{1}\right)$, a subsequence of this subsequence which converges uniformly to a limit $g_{2}(x)$ on $\left(a, c_{2}\right)$, etc. It is clear that $g_{k+1}(x)=g_{k}(x)$ when $a \leqq x \leqq c_{k}$ and that if we define $f_{0}(x)$ as $\lim g_{k}(x)$ when $a \leqq x<b$, then we can by the diagonal process select a subsequence of $f_{n}(x)$ which 
converges uniformly to $f_{0}(x)$ on every closed interval $(a, c)$ for which $c<b$. If we define $f_{0}(b)$ to be $\alpha$, then it is easy to see that $f_{0}(x)$ is in $\mathfrak{B}$ and that

$$
\mu=\lim I\left(f_{n}\right)=I\left(f_{0}\right) .
$$

Hence Theorem 1 is true.

Let $\mathfrak{M}$ be the class of maximizing functions for $I(f)$ in $\mathfrak{B}$; then the result of Theorem 1 is that $\mathfrak{M}$ is not void.

3. A useful identity. Most of our remaining analysis will depend in one way or another on the following result, the proof of which is obvious.

Lemma 2. If $f_{0}(x)$ and $f(x)$ are in $\mathfrak{B}$, if $\eta(x)=f(x)-f_{0}(x)$, and if $A(\eta) \neq 0$, then

$$
\frac{I(f)-I\left(f_{0}\right)}{A(\eta)}=A\left(f_{0}^{-1}\right)-\frac{A\left(f_{0}\right) A\left(\eta / f_{0} f\right)}{A(\eta)}-A\left(\eta / f_{0} f\right) .
$$

We shall use the lemma first to prove the following result.

Lemma 3. If $f_{0}(x)$ is in $\mathfrak{M}$, then $f_{0}(x)$ is continuous on the closed interval $(a, b)$.

Since any function in $\mathfrak{B}$ is continuous when $a \leqq x<b$, it is sufficient to show that $f_{0}(x)$ is continuous when $x=b$. Since $f_{0}(b-)$ exists, suppose that $f_{0}(b-)=\alpha^{\prime}>\alpha$. Choose a positive number $\delta$ for which $\alpha^{\prime}-\delta>\alpha$. Define $\eta(x, \epsilon)$ as 0 when $a \leqq x \leqq b-\epsilon$ and when $x=b$, and so that $f(x, \epsilon)=f_{0}(x)+\eta(x, \epsilon)$ is linear on the open interval $(b-\epsilon, b)$ with limiting end values $f_{0}(b-\epsilon)$ and $\alpha^{\prime}-\delta$. Hence

$$
\alpha^{\prime}-\delta-\beta \leqq \eta(x, \epsilon)<0 \quad(b-\epsilon<x<b) .
$$

Then $f(x, \epsilon)$ is in $\mathfrak{B}$ for sufficiently small $\epsilon$ and so

$$
\left(I(f)-I\left(f_{0}\right)\right) / A(\eta) \geqq 0 .
$$

From the first theorem of the mean for integrals,

$$
A\left(\eta / f_{0} f\right)=A(\eta) / f_{0}\left(x^{*}\right) f\left(x^{*}\right),
$$

in which $b-\epsilon<x^{*}<b$. Since $A(\eta) \rightarrow 0, f_{0}\left(x^{*}\right) \rightarrow \alpha^{\prime}$, and $f\left(x^{*}\right) \rightarrow \alpha^{\prime}-\delta$, it follows from Lemma 2 that

$$
\begin{aligned}
A\left(f_{0}^{-1}\right)-A\left(f_{0}\right) / \alpha^{\prime}\left(\alpha^{\prime}-\delta\right) & \geqq 0, \\
\alpha^{\prime}\left(\alpha^{\prime}-\delta\right) & \geqq A\left(f_{0}\right) B\left(f_{0}\right) .
\end{aligned}
$$

On the other hand, since $f_{0}(x)$ is decreasing, $f_{0}(x) \geqq \alpha^{\prime}$ when $a \leqq x<b$, 
and so

$$
A\left(f_{0}\right) B\left(f_{0}\right) \geqq \alpha^{\prime 2} .
$$

These last two inequalities are incompatible when $\delta>0$, and so Lemma 3 must be true.

4. The behavior of the derivative $f_{0}^{\prime}(x)$ of a maximizing function. We know that $f_{0}^{\prime}(x)$ is a decreasing, nonpositive function and hence the set $E\left(f_{0}\right)$ of its discontinuities on the open set $(a, b)$ is at most denumerable. Our next result is the following lemma.

LEMмa 4. If $f_{0}(x)$ is in $\mathfrak{M}$ then $f_{0}^{\prime}(x)$ is constant on any interval of continuity of $f_{0}^{\prime}(x)$.

If Lemma 4 is false, there exists an interval $(y, z)$ such that $f_{0}^{\prime}(x)$ is continuous when $y<x<z$ and $f_{0}^{\prime}(y+)>f_{0}^{\prime}(z-)$. Therefore, there exists a decreasing sequence $x_{n}$ for which $x_{n} \rightarrow y, f_{0}^{\prime}\left(x_{n+1}\right)>f_{0}^{\prime}\left(x_{n}\right)$. Define $\eta_{n}(x)$ as 0 when $x$ is not on the interval $\left(y, x_{n}\right)$ and so that $f_{n}(x)=f_{0}(x)+\eta_{n}(x)$ is linear on the interval $\left(y, x_{n}\right)$ with end values $f_{0}(y)$ and $f_{0}\left(x_{n}\right)$. If $\eta_{n}(x)$ were identically zero, $f_{0}(x)$ would be linear on the interval $\left(y, x_{n}\right)$, and so $f_{0}^{\prime}\left(x_{n+1}\right)=f_{0}^{\prime}\left(x_{n}\right)$. Hence $\eta_{n}(x)$ does not vanish identically. Moreover, $\eta_{n}(x)<0$ when $y<x<x_{n+1}$ since it is convex on the interval $\left(y, x_{n}\right)$ and vanishes at the endpoints of that interval. The function $f_{n}(x)$ is in $\mathfrak{B}$ and so

$$
\lim _{n \rightarrow \infty} \frac{I\left(f_{n}\right)-I\left(f_{0}\right)}{A\left(\eta_{n}\right)} \geqq 0 .
$$

Moreover,

$$
A\left(\eta_{n} / f_{0} f_{n}\right)=\frac{A\left(\eta_{n}\right)}{f_{0}\left(x_{n}^{*}\right) f_{n}\left(x_{n}^{*}\right)},
$$

in which $y<x_{n}^{*}<x_{n}, A\left(\eta_{n}\right) \rightarrow 0$, and so

$$
\begin{aligned}
A\left(f_{0}^{-1}\right)-A\left(f_{0}\right) /\left[f_{0}(y)\right]^{2} & \geqq 0, \\
{\left[f_{0}(y)\right]^{2} } & \geqq A\left(f_{0}\right) B\left(f_{0}\right) \equiv C^{2} .
\end{aligned}
$$

Now define $\zeta_{n}(x)$ as 0 when $x$ is not in the interval $\left(y, x_{n}\right)$, and so that $g_{n}(x)=f_{0}(x)+\zeta_{n}(x)$ is linear on an interval $\left(y, \gamma_{n}\right)$ with slope $f_{0}^{\prime}(y+)$, is linear on the interval $\left(\gamma_{n}, x_{n}\right)$ with slope $f_{0}^{\prime}\left(x_{n}\right)$, and is continuous on $(a, b)$. Then $y<\gamma_{n}<x_{n}$ and $\zeta_{n}\left(\gamma_{n}\right)>0$, since $f_{0}(x)$ is not linear on the interval $\left(y, x_{n}\right)$. Moreover $\zeta_{n}(x) \geqq 0$ since $f_{0}(x)$ is concave and hence $\zeta_{n}(x)>0$ when $y<x<x_{n}$. The function $g_{n}(x)$ is in $\mathfrak{B}$ and so 


$$
\lim _{n \rightarrow \infty} \frac{I\left(g_{n}\right)-I\left(f_{0}\right)}{A\left(\zeta_{n}\right)} \leqq 0 .
$$

This limit is evaluated exactly as in the preceding paragraph, and we deduce that

$$
\left[f_{0}(y)\right]^{2} \leqq A\left(f_{0}\right) B\left(f_{0}\right) \equiv C^{2} .
$$

We conclude from the inequalities (3) and (4) that $f_{0}(y)=C$. It is clear that we could use the same arguments at $z$ and deduce also that $f_{0}(z)=C$. Since $f_{0}(x)$ is decreasing, we must then have that $f_{0}(x)=C$ when $y \leqq x \leqq z$, and so $f_{0}^{\prime}(x)=0=f_{0}^{\prime}(y+)=f_{0}^{\prime}(z-)$. From this contradiction we infer the truth of Lemma 4.

5. The set $E\left(f_{0}\right)$ of discontinuities of $f_{0}^{\prime}(x)$. We are going to show ultimately that the set $E\left(f_{0}\right)$ consists of precisely one point for any function $f_{0}(x)$ in $\mathfrak{M}$. The first step in this demonstration is the following result.

Lemma 5. If $f_{0}(x)$ is in $\mathfrak{M}$, then the set $E\left(f_{0}\right)$ is not void.

If $E\left(f_{0}\right)$ is void, then $(a, b)$ is an interval of continuity of $f_{0}^{\prime}(x)$ and so $f_{0}^{\prime}(x)$ is constant on $(a, b)$. This constant must be

$$
(\beta-\alpha) /(a-b) \text {, }
$$

and so

$$
\begin{aligned}
& f_{0}(x)=\beta-\frac{(\beta-\alpha)(x-a)}{(b-a)}, \\
& I\left(f_{0}\right)=\frac{(\alpha+\beta) \ln (\beta / \alpha)}{2(\beta-\alpha)} .
\end{aligned}
$$

Let us define $f(x, \epsilon)$ so that

$$
f(x, \epsilon)= \begin{cases}\beta & (a \leqq x \leqq a+\epsilon), \\ \beta-(\beta-\alpha)(x-a-\epsilon) /(b-a-\epsilon) & (a+\epsilon \leqq x \leqq b) .\end{cases}
$$

Then $f(x, \epsilon)$ is in $\mathfrak{B}$ and

$$
\begin{aligned}
I(f)= & I\left(f_{0}\right)+\frac{\epsilon}{2(b-a)}\left(\frac{1+\xi}{\xi}-\frac{2 \ln \xi}{\xi-1}\right) \\
& +\frac{\epsilon^{2}}{2(b-a)^{2}}\left(\frac{\xi-1}{\xi}-\ln \xi\right),
\end{aligned}
$$

in which $\xi=\beta / \alpha>1$. It is easy to verify that the coefficient of 
$\epsilon / 2(b-a)$ is positive when $\xi>1$ and hence $I(f)>I\left(f_{0}\right)$ for sufficiently small positive $\epsilon$. From this contradiction we infer the truth of Lemma 5.

Lemma 6. If $f_{0}(x)$ is in $\mathfrak{M}$ and if $y$ is any point in the set $E\left(f_{0}\right)$ then

$$
f_{0}(y) \geqq C=\left\{A\left(f_{0}\right) B\left(f_{0}\right)\right\}^{1 / 2} .
$$

We define $\eta(x, \epsilon)$ as 0 when $|x-y| \geqq \epsilon$ and so that $f(x, \epsilon)=f_{0}(x)$ $+\eta(x, \epsilon)$ is linear on the interval $(y-\epsilon, y+\epsilon)$ and continuous on $(a, b)$. Then $\eta(x, \epsilon)<0$ when $y-\epsilon<x<y+\epsilon, f(x, \epsilon)$ is in $\mathscr{B}$, and so

$$
\lim _{\epsilon \rightarrow 0} \frac{I(f)-I\left(f_{0}\right)}{A(\eta)} \geqq 0 .
$$

The limit may be evaluated exactly as in the proof of Lemma 4, and leads immediately to the inequality (6).

LemMa 7. If $f_{0}(x)$ is in $\mathfrak{M}$ and if $y$ is any point in the set $E\left(f_{0}\right)$ for which there exists another point $z$ in $E\left(f_{0}\right)$ such that $z<y$ and the interval $(z, y)$ contains no other points of $E\left(f_{0}\right)$, then

$$
f_{0}(y)=C=\left\{A\left(f_{0}\right) B\left(f_{0}\right)\right\}^{1 / 2} \text {. }
$$

Choose $\lambda$ so that $f_{0}^{\prime}(z+)<\lambda<f_{0}^{\prime}(z-)$. Define $\eta(x, \lambda)$ as 0 when $a \leqq x \leqq z$ and when $y \leqq x \leqq b$ and so that $f(x, \lambda)=f_{0}(x)+\eta(x, \lambda)$ is linear on an interval $(z, \gamma)$ with slope $\lambda$, is linear on the interval $(\gamma, y)$ with slope $f_{0}^{\prime}(y+)$, and is continuous on $(a, b)$. Since $f_{0}(x)$ is linear on the interval $(z, y)$ with slope $f_{0}^{\prime}(z+)$, we have that $z<\gamma<y$ and that $\eta(x, \lambda)>0$ when $z<x<y$. Hence

$$
\lim _{\lambda \rightarrow f_{0}^{\prime}(z+)} \sup _{(2+)} \frac{I(f)-I\left(f_{0}\right)}{A(\eta)} \leqq 0 .
$$

Since $\eta(x, \lambda) \geqq 0$ and both $f_{0}$ and $f$ are decreasing

$$
-A\left(\eta / f_{0} f\right) \geqq-A(\eta) /\left[f_{0}(y)\right]^{2} .
$$

Since $A(\eta) \rightarrow 0$, we deduce that

$$
\begin{aligned}
0 & \geqq A\left(f_{0}^{-1}\right)-A\left(f_{0}\right) /\left[f_{0}(y)\right]^{2}, \\
f_{0}(y) & \leqq C .
\end{aligned}
$$

Since Lemma 6 holds, we conclude that Lemma 7 is true.

LEMMA 8. If $f_{0}(x)$ maximizes $I(f)$ on $\mathfrak{B}$, and if $y$ is any point in the set $E\left(f_{0}\right)$ for which there exists an increasing sequence $z_{n}$ of points of $E\left(f_{0}\right)$ which converge to $y$, then $f_{0}(y)=C=\left\{A\left(f_{0}\right) B\left(f_{\theta}\right)\right\}^{1 / 2}$. 
Define $\eta_{n}(x)$ as 0 when $a \leqq x \leqq z_{n}$ and $y \leqq x \leqq b$, and so that $f_{n}(x)$ $=f_{0}(x)+\eta_{n}(x)$ is linear on an interval $\left(z_{n}, \gamma_{n}\right)$ with slope $f_{0}^{\prime}\left(z_{n}-\right)$, is linear on the interval $\left(\gamma_{n}, y\right)$ with slope $f_{0}^{\prime}(y+)$, and is continuous on $(a, b)$. Then $z_{n}<\gamma_{n}<y$ and $\eta_{n}(x)>0$ when $z_{n}<x<y$ since a corner $z_{n+1}$ occurs on the open interval $\left(z_{n}, y\right)$. Hence

$$
\begin{array}{r}
A\left(f_{0}^{-1}\right)-\frac{A\left(f_{0}\right)}{\left[f_{0}(y)\right]^{2}}=\lim _{n \rightarrow \infty} \frac{I\left(f_{n}\right)-I\left(f_{0}\right)}{A\left(\eta_{n}\right)} \\
f_{0}(y) \leqq 0,
\end{array}
$$

Since Lemma 6 holds, we conclude that Lemma 8 is true.

Lemma 9. If $f_{0}(x)$ is in $\mathfrak{M}$, then the set $E\left(f_{0}\right)$ has at most two points.

Suppose on the contrary that $E\left(f_{0}\right)$ has three distinct points $u<v<w$. Then either Lemma 7 or Lemma 8 applies to the points $v$ and $w$ and so $f_{0}(v)=f_{0}(w)=C$. Since $f_{0}(x)$ is decreasing, $f_{0}(x)=C$ when $v<x<w, f_{0}^{\prime}(v+)=0$. On the other hand, $f_{0}^{\prime}(x)$ is a nonpositive decreasing function and $f_{0}^{\prime}(v+)<f_{0}^{\prime}(v-) \leqq 0$. From this contradiction we infer that Lemma 9 is true.

Lemma 10. If $f_{0}(x)$ is in $\mathfrak{M}$, and if the set $E\left(f_{0}\right)$ has exactly two points $y<z$, then $f_{0}(y)=\beta, f_{0}(z)=C$. If $E$ has exactly one point $y$, then either $f_{0}(y)=\beta$ or $f_{0}(y)=C=\left\{A\left(f_{0}\right) B\left(f_{0}\right)\right\}^{1 / 2}$.

Suppose in either case that $f_{0}(y)<\beta$. Then define $\eta(x, \epsilon)$ as 0 when $x \geqq y$ and so that $f(x, \epsilon)=f_{0}(x)+\eta(x, \epsilon)$ is linear on the interval $(y-\epsilon, y)$ with slope $f_{0}^{\prime}(y+)$, is linear on the interval $(a, y-\epsilon)$, assumes the value $\beta$ when $x=a$, and is continuous on $(a, b)$. Then $\eta(x, \epsilon)>0$ when $0<x<y, A(\eta) \rightarrow 0$ with $\epsilon$, and $f(x, \epsilon)$ is in $\mathfrak{B}$ for sufficiently small positive $\epsilon$. Hence

$$
-A\left(\eta / f_{0} f\right) \geqq-A(\eta) /\left[f_{0}(y)\right]^{2} .
$$

Since $A(\eta) \rightarrow 0$,

$$
0 \geqq \lim \sup \frac{I(f)-I\left(f_{0}\right)}{A(\eta)} \geqq A\left(f_{0}^{-1}\right)-\frac{A\left(f_{0}\right)}{\left[f_{0}(y)\right]^{2}},
$$

and so $f_{0}(y) \leqq C$. Since Lemma 6 holds, $f_{0}(y)=C$. This is sufficient to prove the second sentence of Lemma 10 . If $E\left(f_{0}\right)$ has another point $z>y$, then $f_{0}(z)=C$ also since Lemma 7 holds. Hence $f_{0}(x)=C$ when $y \leqq x \leqq z, f_{0}^{\prime}(y+)=0$, and this is impossible. From this contradiction we infer the truth of the first sentence of Lemma 10.

Lemma 11. If $f_{0}(x)$ is in $\mathfrak{M}$, then the set $E\left(f_{0}\right)$ has exactly one point. 
Suppose on the contrary that $E\left(f_{0}\right)$ has exactly two distinct points $y<z$. Then from Lemma $10, f_{0}(y)=\beta, f_{0}(z)=C$ and $f_{0}(x)$ is linear on the intervals $(a, y),(y, z)$ and $(z, b)$. Define $f(x, \epsilon)$ to be $f_{0}(x)$ on the intervals $(a, y)$ and $(z, b)$, linear with slope $f_{0}^{\prime}(z+)$ on the interval $(z-\epsilon, z)$, linear on the interval $(y, z-\epsilon)$, and continuous on $(a, b)$. Define

$$
\Delta=\frac{(z-y)}{2(b-a)}\left[f_{0}^{\prime}(z-)-f_{0}^{\prime}(z+)\right] .
$$

Then $\Delta>0$, and

$$
\begin{aligned}
A(f) & =A\left(f_{0}^{\prime}\right)+\epsilon \Delta, \\
A\left(f^{-1}\right) & =A\left(f_{0}^{-1}\right)+\epsilon \Delta \Delta_{1}+O\left(\epsilon^{2}\right)
\end{aligned}
$$

in which

$$
\Delta_{1}=\frac{2}{\beta-C}\left\{\frac{\ln (\beta / C)}{\beta-C}-\frac{1}{C}\right\} .
$$

Hence

$$
I(f)=I\left(f_{0}\right)+\epsilon \Delta\left\{A\left(f_{0}^{-1}\right)+A\left(f_{0}\right) \Delta_{1}\right\}+O\left(\epsilon^{2}\right),
$$

and so the coefficient of $\epsilon \Delta$ must be nonpositive. On the other hand, since $C^{2}=A\left(f_{0}\right) B\left(f_{0}\right)$,

$$
A\left(f_{0}^{-1}\right)+A\left(f_{0}\right) \Delta_{1}=\frac{A\left(f_{0}\right)}{C^{2}}\left\{1+\frac{2}{\zeta-1}\left(\frac{\ln \zeta}{\zeta-1}-1\right)\right\}
$$

in which $\zeta=\beta / C>1$. The quantity within the braces is always positive when $\zeta>1$, and from this contradiction we deduce the truth of Lemma 11.

LEMмA 12. If $f_{0}(x)$ is in $\mathfrak{M}$ the value $f_{0}(y)$ at the unique discontinuity $y$ of $f_{0}^{\prime}(x)$ is $\beta$.

Suppose on the contrary that $f_{0}(y)<\beta$. According to Lemma 10 , $f_{0}(y)=C$. Let $f(x, \epsilon)$ be defined as in the proof of Lemma 10. Then

$$
\begin{aligned}
A(f) & =A\left(f_{0}\right)+\epsilon \Delta_{2}, \\
A\left(f^{-1}\right) & =A\left(f_{0}^{-1}\right)+\epsilon \Delta_{2} \Delta_{1}+O\left(\epsilon^{2}\right),
\end{aligned}
$$

in which

$$
\Delta_{2}=\frac{(y-a)}{2(b-a)}\left[f_{0}^{\prime}(y-)-f_{0}^{\prime}(y+)\right]>0 .
$$


Hence

$$
I(f)=I\left(f_{0}\right)+\epsilon \Delta_{2}\left\{A\left(f_{0}^{-1}\right)+A\left(f_{0}\right) \Delta_{1}\right\}+O\left(\epsilon^{2}\right) .
$$

Since the coefficient of $\epsilon \Delta_{2}$ is the same quantity encountered in the proof of Lemma 11, it is positive and so $I(f)>I\left(f_{0}\right)$ for sufficiently small positive $\epsilon$. From this contradiction we infer the truth of Lemma 12 .

6. The maximizing function $f_{0}(x)$. We are now in a position to prove our principal result.

TheOREM 2. If $f(x)$ is in $\mathfrak{B}$, then

$$
1 \leqq I(f) \leqq I\left(f_{0}\right)
$$

in which

$$
\begin{aligned}
f_{0}(x) & = \begin{cases}\beta & (a \leqq x \leqq y), \\
\beta-(\beta-\alpha)(x-y) /(b-y) & (y \leqq x \leqq b),\end{cases} \\
y & =a+\frac{(b-a)((1+\xi) / \xi-2 \ln \xi /(\xi-1))}{2(\ln \xi-(\xi-1) / \xi)}
\end{aligned}
$$

As a consequence of the preceding lemmas, we know that any maximizing function $f_{0}(x)$ must be of the form described in Theorem 2 for some value $y$. If $y=a+\epsilon$ we have already $I\left(f_{0}\right)$ in equation (5).

This equation may be written as

$$
I\left(f_{0}\right)=L+M u+N u^{2},
$$

in which

$$
\begin{aligned}
L & =\frac{(\xi+1) \ln \xi}{2(\xi-1)}, \\
M & =\frac{1}{2}\left(\frac{1+\xi}{\xi}-\frac{2 \ln \xi}{\xi-1}\right)>0, \\
V & =\frac{1}{2}\left(\frac{\xi-1}{\xi}-\ln \xi\right)<0, \\
u & =(y-a) /(b-a), \\
\xi & =\beta / \alpha .
\end{aligned}
$$

This function of $u$ is concave and attains its maximum value when

$$
u=-M / 2 N \text {. }
$$

Since $M>0, N<0$ and $2 N+M<0$, this value of $u$ lies in the open 
interval $(0,1)$ and yields the value of $y$ specified in the theorem. The maximum value is $\left(4 L N-M^{2}\right) / 4 N$, and this value is the one specified in the inequality (2).

Hence the only maximizing function possible is the one specified in Theorem 2. Since a maximizing function is known to exist, this function must be a maximizing function and in fact the only maximizing function.

A short table of values of the upper bounds in (1) and (2) as a function of the ratio $\beta / \alpha$ is given below.

\begin{tabular}{ccc}
\hline \hline$\beta / \alpha$ & upper bound on $\mathfrak{A}$ & upper bound on $\mathfrak{B}$ \\
\hline 1.0 & 1.0000 & 1.0000 \\
1.5 & 1.0417 & 1.0171 \\
2.0 & 1.1250 & 1.0481 \\
2.5 & 1.2250 & 1.0816 \\
3.0 & 1.3333 & 1.1146 \\
\hline
\end{tabular}

\section{REFERENCES}

1. P. G. Laurson and W. J. Cox, Mechanics of materials, Wiley, 1938, pp. 345-349.

2. W. H. McAdams, Heat transmission, McGraw-Hill, 1954, pp. 11-19.

3. G. Polya and G. Szegö, Aufgaben und Lehrsätze der Analysis, vol. I, Dover, 1945, pp. 57 and 214.

Nuclear Development Associates, Inc. 